\section{Moyamoya in Hispanics: not only in Japanese}

\author{
Sarmad Said, Chad J. Cooper, \\ Haider Alkhateeb, Juan M. Galvis, \\ German T. Hernandez, Hasan J. Salameh \\ Department of Internal Medicine, Paul L. \\ Foster School of Medicine, Texas Tech \\ University Health Sciences Center, EI \\ Paso, TX, USA
}

\section{Abstract}

Moyamoya disease was first described in 1957 as hypoplasia of the bilateral internal carotid arteries, the characteristic appearance of the associated network of abnormally dilated collateral vessels on angiography was later likened to something hazy, like a puff of cigarette smoke, which, in Japanese, is moyamoya. This paper describes two cases of moyamoya presentations, including moyamoya disease and moyamoya syndrome. Moyamoya may rarely occur in North American Hispanic patients. The presentation can vary significantly and ranges bwtween fulminant outcome and prolonged survival. Awareness about moyamoya and its different presentations may be beneficial for the patients and can improve the outcome.

\section{Introduction}

Moyamoya disease (MMD), translated in Japanese as hazy or puffy, is a chronic progressive occlusive arteriopathy of the anterior cerebral circulation. The vascular pathology of MMD causes large intracranial artery occlusion and collateral circulation leading to acute infarcts, which have a higher tendency to develop recurrent strokes causing a significant cognitive impairment and neurologic decline.1,2 Overall incidence of Moyamoya is 0.086 cases per 100,000 people. MMD has the highest prevalence among Japanese children $(6.03$ per 100,000$)$. An increase in the prevalence of Moyamoya has been demonstrated among non-Asian populations. The reported occurrence in the Caucasian population in the US is the highest compared to other populations, but Caucasians represent $72.4 \%$ of the US population. In general, Moyamoya disease is observed among the various races based on their proportions of the total US population. ${ }^{3}$

The occurrence of the disease shows a bimodal age distribution at 5-9 years and 35-50 years with female predominance. ${ }^{4}$ The exact etiology of MMD is unknown, however the increased familial incidence suggests a genetic cause. Accumulating evidence suggests that the RNF213 gene on chromosome $17 \mathrm{q} 25.3$ is an important susceptibility factor for MMD in East Asian populations. 5,6 In a report from Japan, the c. $14576 \mathrm{G}>\mathrm{A}$ variant of RNF213 was found in $95 \%$ of 41 patients with familial MMD, $79 \%$ of 163 patients with sporadic MMD, and $2 \%$ of 283 normal control subjects. ${ }^{6}$ Other studies have linked familial Moyamoya disease to chromosomes 3p24.2-p26, 6q25, 8q23, and 12p12.7,8 Familial cases of MMD in the US are approximately $2 \%$, but in Japan a family history of Moyamoya disease is present in $12.1 \%$ of patients. Environmental factors can be considered as contributing factors to this observation. 3,9

Especially in North American patients the clinical presentation can be atypical with a later onset and may cause a delay of the diagnosis. These patients are less likely to have hemorrhagic stroke.10,11 Kuroda et al. published in 2005 an article about the clinical features of MMD in adults indicating that the disease has a high rate of progression, even in asymptomatic patients, and medical alone cannot stop the disease progression. ${ }^{12}$ Over a period of 5 years about 70\% of the MMD-patients experience a symptomatic progression; without a treatment the disease outcome is poor. ${ }^{13}$ In contrast surgical intervention may be more beneficial. A meta-analysis of 2005 on pediatric patients involving 1156 subjects showed that symptomatic disease progression occurred only in $2.6 \%$ of the patients after surgery. 14

We describe a case series of advanced Moyamoya disease in a Hispanic male and protracted Moyamoya syndrome in a Hispanic female with an acute, fulminant and protracted progression, respectively.

\section{Case Report \#1}

A 42-year-old Hispanic male without significant past medical history presented with a 4week history of slow mentation, difficulty concentrating, and change in personality. He complained of bilateral lower extremity weakness, as well as a presyncopal episode and sudden onset of numbness involving his right body side 3 days before admission. Other symptoms included intermittent nausea and vomiting. Physical examination included slow mentation, impaired concentration, facial weakness of the right lower half, dysarthria, and mild weakness in the lower extremities without sensory level or sphincter dysfunction. Full strength in his upper extremities, diminished reflexes throughout, and intact sensation were noted. No cerebellar dysfunction, nystagmus, or Babinski signs were present. Laboratory
Correspondence: Hasan J. Salameh, Department of Internal Medicine, Paul L. Foster School of Medicine, Texas Tech University Health Sciences Center, 4800 Alberta Avenue, El Paso, 79905 TX, USA.

Tel.: +1.915.443.5505 - Fax: +1.915.545.6634

E-mail: hasan.salameh@ttuhsc.edu

Key words: moyamoya disease, moyamoya syndrome, stroke, Hispanic population.

Contributions: SS took care of the patient and arranged all required medical steps, wrote the draft of the manuscript; CC collected literature and reviewed the final version of the manuscript; HA took care of the patient and contributed in updating the team, he reviewed the final version, edited and approved it; JMG, GTH, analyzed the manuscript and performed independent proofreading; HJS, controlled all the above-mentioned steps and made the final medical decisions.

Conflict of interests: the authors declare no potential conflict of interests.

Received for publication: 24 February 2014

Revision received: 25 March 2014.

Accepted for publication: 28 March 2014.

This work is licensed under a Creative Commons Attribution NonCommercial 3.0 License (CC BYNC 3.0).

(C) Copyright S. Said et al., 2014

Licensee PAGEPress, Italy

Neurology International 2014; 6:5369

doi:10.4081/ni.2014.5369

tests for systemic autoimmune, inflammatory, and infectious diseases, and neoplasm were all negative.

Initial unenhanced head computed tomography (CT) showed hypodense lesions in the body and genu of the corpus callosum and right basal ganglia. A subsequent brain magnet-resonance-imaging revealed multiple supratentorial lesions involving the rostrum of the corpus callosum, genu of the right internal capsule and basal ganglia and subcortical cingulate white matter, with variable degrees of enhancement associated with mass effect (Figure 1). Initial differential diagnoses included an acute demyelinating process versus primary CNS lymphoma, glioma, or acute/subacute ischemic infarct.

Cerebrospinal fluid analysis (CSF) showed mild increase of total protein and elevated CSF levels of myelin basic protein, without oligoclonal bands. High doses of intravenous methylprednisolone ( $1 \mathrm{gm} /$ day) were administered; 72 hours after admission, the patient developed altered level of consciousness, aphasia, left gaze deviation, and right hemiplegia.

Cerebral angiography (Figure 2) demonstrated bilateral intracranial arteriopathy with occlusion of the supraclinoid segment of the 
left intracranial internal carotid artery distal to the takeoff of the left anterior choroidal artery, occlusion of the right anterior cerebral artery, and severe disease in the proximal right middle cerebral artery. The distal branches of the right middle and anterior cerebral arteries were mainly fed by collateral anastomoses from the anterior and posterior choroidal arteries, producing arterial blush (puff of smoke) consistent with an angiographic Moyamoya pattern.

Within few days the patient developed a massive infarction involving almost the entire left hemisphere and the bilateral anterior cerebral arterial territories, associated cerebral edema, midline shift complicated by brain herniation, and eventually death 11 days after admission.

\section{Case Report \#2}

A 29-year old Hispanic female with a significant past medical history for repaired patent foramen ovale, pulmonary valve stenosis and right ventricular outflow obstruction, which were both surgically treated 2 years ago presented with 3-days history of severe bifrontal headache and altered mental status. Physical examination and laboratory workup were unremarkable. Head-CT demonstrated subarachnoid hemorrhage seen throughout basilar cisterns including suprasellar, interpeduncular, perimesencephalic, and prepontine cisterns. Head-CT-angiography (CTA) showed bilateral intracranial internal carotid artery (ICA) occlusion with concentric luminal narrowing of the cervical ICAs, diffuse intracranial proliferative arteriopathy in the bilateral territories of the anterior and middle cerebral arteries and ruptured saccular aneurysm arising from the right posterior cerebral artery. Cerebral angiogram confirmed these findings and a coiling embolization of a ruptured 3.5 $\mathrm{mm}$ high flow aneurysm arising from the distal aspect of the basilar artery was performed. The angiogram also demonstrated bilateral severe stenosis of the internal carotid arteries with multiple transdural collaterals consistent with moyamoya angiographic pattern (Figure $3)$. The neurosurgery service was consulted, however the patient refused further surgical evaluation and was discharged on the $7^{\text {th }}$ day of hospitalization. Unfortunately the patient did not follow up with neuroradiology and neurosurgery and presented 15 months later with acute onset of altered level of consciousness. She was intubated in the emergency department, placed on mechanical ventilation and admitted with a Glasgow Coma Scale (GCS) of 6 to the medical intensive care unit. The repeat-head-CT showed extensive hemorrhage confined to the choroid plexus bilaterally involving the third and forth ventricles resulting in a left-to-right $4 \mathrm{~mm}$ midline shift with moderate hydrocephalus. On the following day her GCS declined to 3 and her cardiac rhythm changed to bradycardia and later asystole. Cardiopulmonary resuscitation was unsuccessful and the patient died on the following day of hospitalization.

\section{Discussion}

Moyamoya disease is an idiopathic progressive intracranial occlusive arteriopathy, which can be complicated with ischemic and hemorrhagic cerebrovascular events. The angiographic feature of collateralization is pathognomonic for Moyamoya pattern. The Moyamoya syndrome can be associated with severe intracranial atherosclerotic disease, sickle cell disease, neurofibromatosis type I, cranial radiation therapy, and trisomy 21.15 Typically moyamoya disease and moyamoya syndrome present during childhood, however the incidence of both forms in North America occurs in the mid-40s. ${ }^{3}$

Diagnostic criteria for idiopathic moyamoya include the following major requirements: stenosis or occlusion at the terminal portion of the internal carotid artery and at the proximal portion of the anterior and middle cerebral arteries on MRA or conventional cerebral angiography; abnormal vascular networks in the basal ganglia on MRA; angiographic findings are present bilaterally. ${ }^{16}$

Magnet-resonance imaging is the preferred radiological modality. Contrast-enhanced T1weighted images and FLAIR (fluid-attenuated inversion recovery) images can show a linear pattern of increased signal in the leptomeninges and perivascular spaces, known as the ivy sign. Transcranial Doppler ultrasonography (TCD) can be used to evaluate large artery stenosis and intracranial hemodynamics by measuring blood flow velocity of the large intracranial vessels at the circle of Willis. SPECT can also be used to demonstrate the decrease blood and oxygen supply to areas of the brain. ${ }^{17,18}$

Overall, the acute treatment goals are to reduce intracranial pressure, improve cerebral blood flow and control any seizures. Ventricular drainage and/or hematoma removal may be required. Thrombolytic therapy in the event of ischemic stroke is not recommended, since adults usually present with a hemorrhagic stroke as the collaterals have a tendency to rupture. 19

Surgical revascularization is advised in both

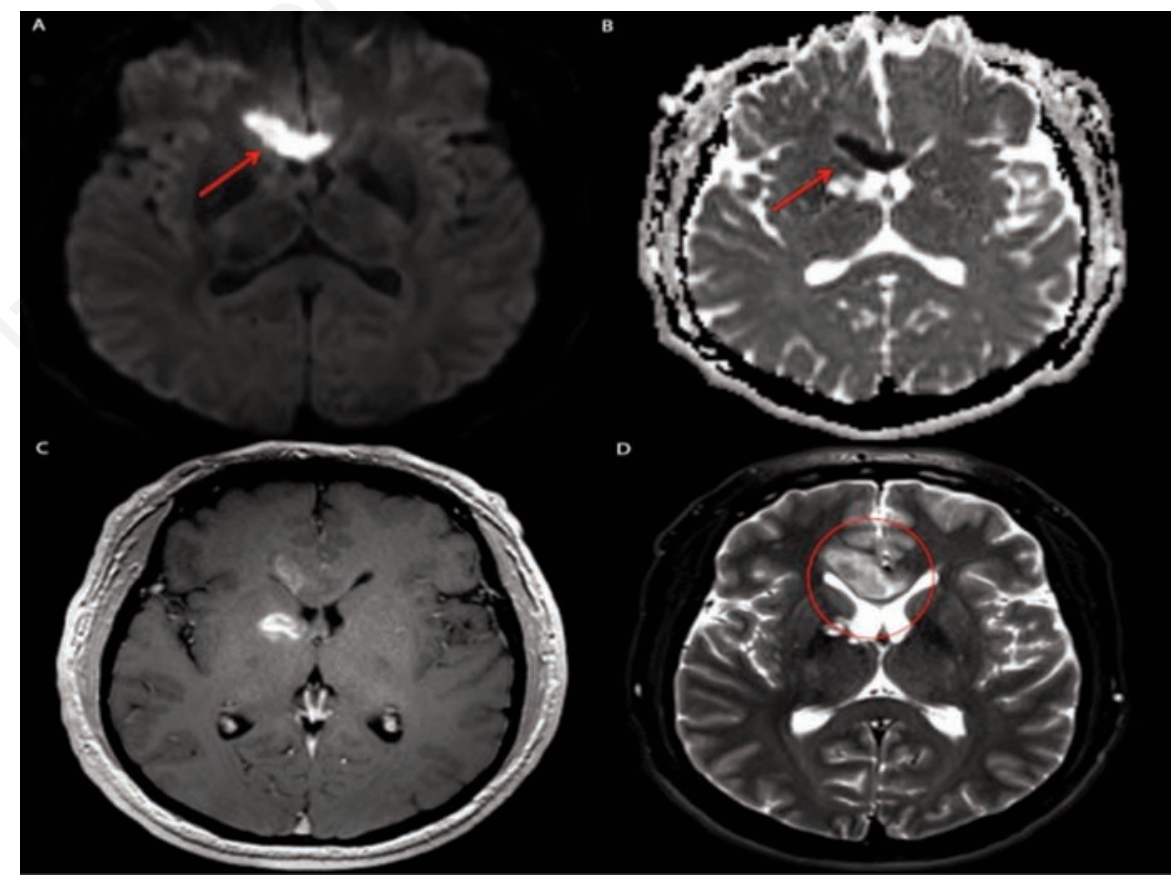

Figure 1. Case \#1. A) and B) diffusion weighted sequence and apparent diffusion coefficient map, respectively, demonstrate a focal area of restriction of diffusion involving the right rostrum, genu and body of the Corpus Callosum with mild enhancement on postgadolinium T1W sequence (C) and moderate edema and local mass effect on T2W sequence (D). A second lesion involving the right basal ganglia and genu of the right internal capsule demonstrates incomplete peripheral enhancement with central necrosis, without mass effect or restriction of diffusion. 

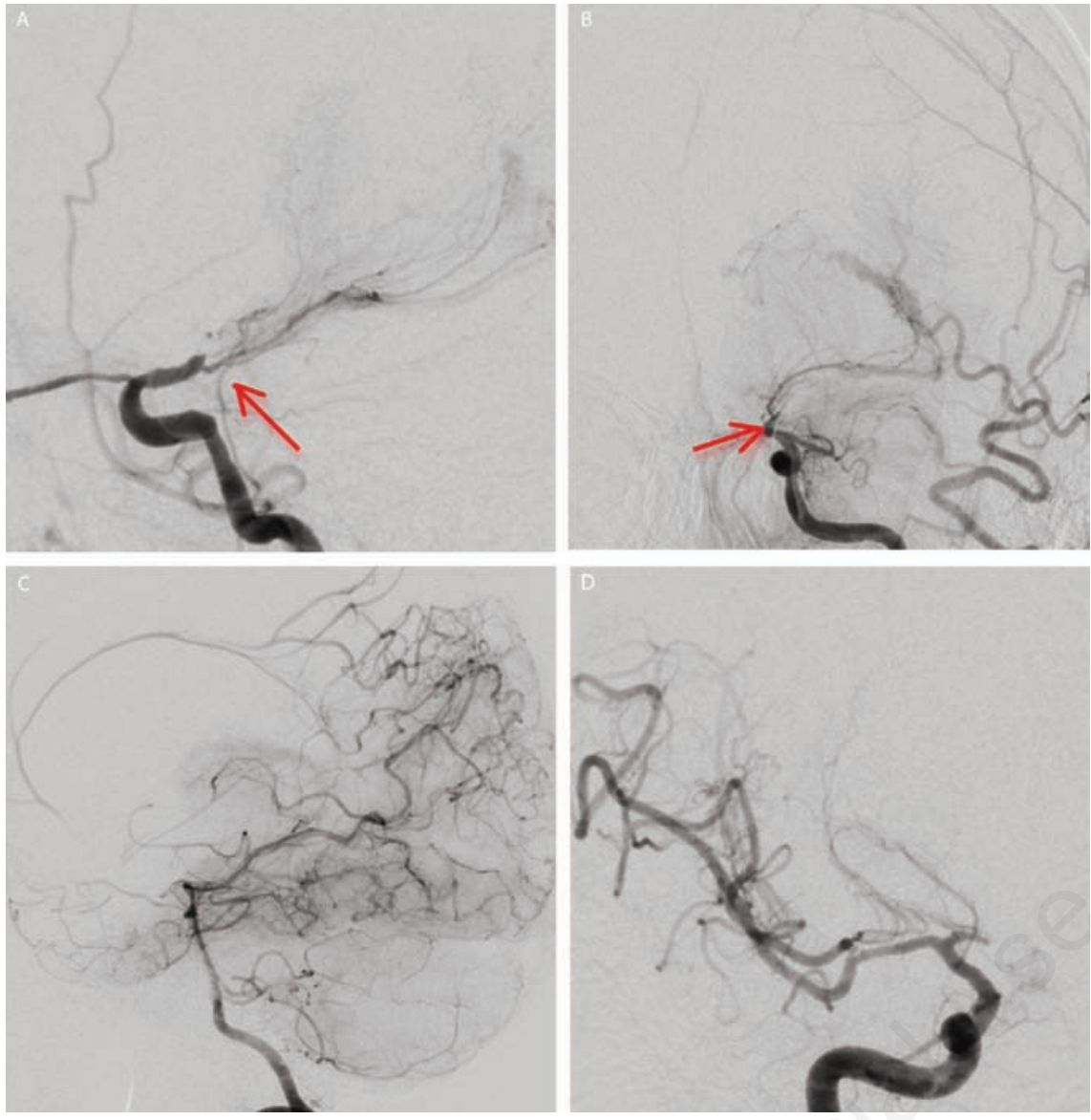

Figure 2. Case \#1. Biplanar cerebral angiography of the left internal carotid artery (A and $B$, lateral and AP respectively) demonstrated complete occlusion of the left supraclinoid internal carotid artery with a patent left anterior choroidal artery supplying lenticulostriatal vessels giving the angiographic appearance of a I, suggestive of Moyamoya angiographic pattern. Collateral flow from the right posterior choroidal artery supplying the pericollosal, frontopolar and callosmarginal arteries demonstrated on lateral view on selective angiogram of the left vertebral artery $(C)$. Intracranial view of the right internal carotid artery in the AP projection (D) demonstrates an irregular M1 segment and complete occlusion of the mid A1 segment of anterior cerebral artery. Cortical branches also appeared irregularity consistent with a proliferative intracranial vasculopathy.

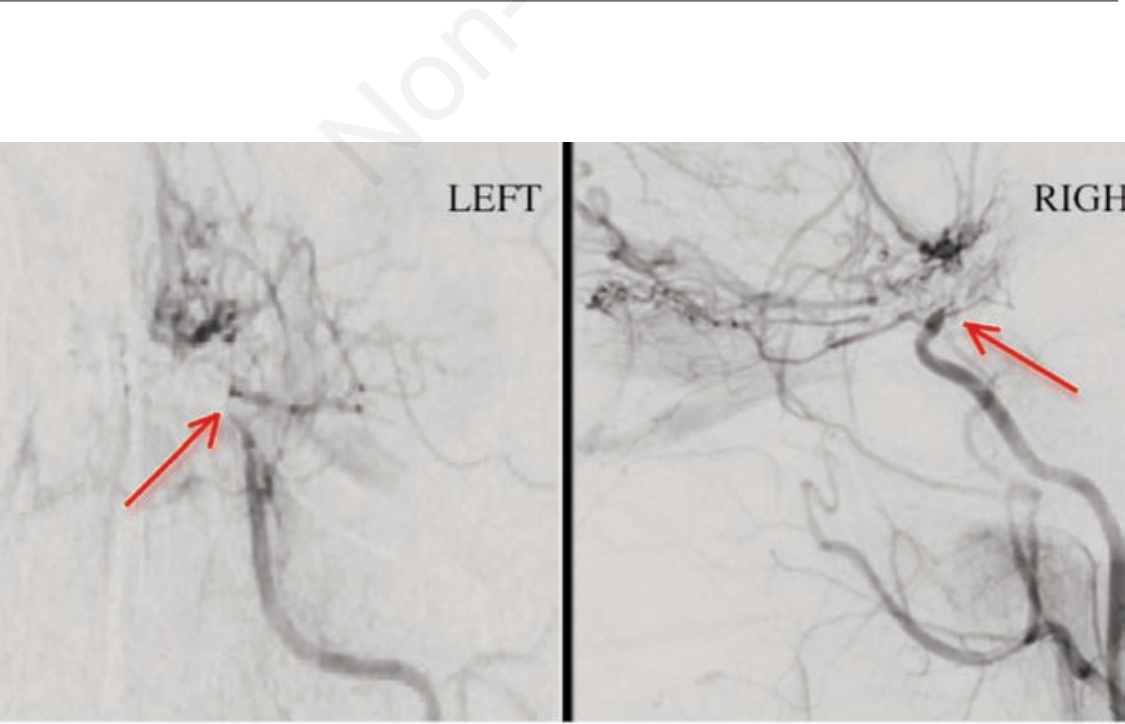

Figure 3. Case \#2. Bilateral severe stenosis (near occlusion) of intracranial supraclinoid internal carotid arteries (left worse than right) with multiple transdural basilar collaterals consistent with moyamoya angiographic pattern. children and adults presenting with progressive symptoms and/or cognitive decline. There are multiple surgical options, but the treatment of choice is thought to be a direct superficial temporal artery to middle cerebral artery bypass (external carotid-internal carotid bypass surgery). ${ }^{20}$ Other surgical modalities including EDAS (encephaloduroarteriosynangiosis), EMS (encephalomyosynangiosis), and the multiple burr holes procedure are available, however the outcome remains controversial.

\section{Conclusions}

We describe an uncommon variety of moyamoya presentation in the setting of moyamoya disease with a fulminant progression (case 1) and moyamoya syndrome with delayed presentation but also with fatal outcome (case 2).

Despite the limited prognosis and the evidences known about the disease progression a fulminant course with multiple bilateral large lobar infarcts precipitating herniation and death within days is uncommon (case 1). In contrast subarachnoid hemorrhage as a result of saccular aneurysmatic formation in the setting of moyamoya syndrome is more common. However neurointerventional procedures may not hold the disease progression but can prolong survival (case 2). The outcome in both settings remains unfortunately the same.

While definitive treatment in the acute fulminant phase may be futile and not indicated, ascertaining the correct diagnosis may help provide a more accurate prognosis.

\section{References}

1. Derdeyn CP. Moyamoya disease and moyamoya syndrome. N Engl J Med 2009; 361:97.

2. Said S, Cooper CJ, Chowdhury F, et al. A case with unusual stroke and fulminant outcome in a hispanic male. Am J Case Rep 2013;14:424-9.

3. Kainth D, Chaudhry SA, Kainth $H$. Epidemiological and clinical features of moyamoya disease in the USA. Neuroepidemiology 2013;40:282-7.

4. Duan L, Bao XY, Yang WZ. Moyamoya disease in China: its clinical features and outcomes. Stroke 2012;43:56.

5. Miyatake S, Miyake N, Touho $\mathrm{H}$. Homozygous c.14576G $>\mathrm{A}$ variant of RNF213 predicts early-onset and severe form of moyamoya disease. Neurology 2012;78:803.

6. Mineharu Y, Liu W, Inoue K. Autosomal 
dominant moyamoya disease maps to chromosome 17q25.3. Neurology 2008;70:2357.

7. Sakurai K, Horiuchi Y, Ikeda H. A novel susceptibility locus for moyamoya disease on chromosome $8 \mathrm{q} 23$. J Hum Genet 2004;49:278.

8. Yilmaz EY, Pritz MB, Bruno A. Moyamoya: Indiana university medical center experience. Arch Neurol 2001;58:1274.

9. Kuriyama S, Kusaka Y, Fujimura M. Prevalence and clinicoepidemiological features of moyamoya disease in Japan. Stroke 2008;39:42-7.

10. Chiu D, Shedden P, Bratina P, Grotta JC. Clinical features of moyamoya disease in the United States. Stroke 1998;29:1347.

11. Andreadou E, Papadimas GK, Sifakis N, Sfagos C. Corpus callosum infarct associated with combined variants in circle of willis. Neurol India 2010;58:785-6.

12. Kuroda S, Ishikawa T, Houkin K. Incidence and clinical features of disease progres- sion in adult moyamoya disease. Stroke 2005;36:2148-53.

13. Choi JU, Kim DS, Kim EY, Lee KC. Natural history of moyamoya disease: comparison of activity of daily living in surgery and non surgery groups. Clin Neurol Neurosurg 1997;99:S11-8.

14. Fung LW, Thompson D, Ganesan V. Revascularisation surgery for paediatric moyamoya: a review of the literature. Childs Nerv Syst 2005;21:358-64.

15. Scott RM, Smith ER. Moyamoya disease and moyamoya syndrome. N Engl J Med 2009;360:1226-37.

16. Fukui M. Guidelines for the diagnosis and treatment of spontaneous occlusion of the circle of Willis ('moyamoya' disease). Research Committee on Spontaneous Occlusion of the Circle of Willis (Moyamoya Disease) of the Ministry of Health and Welfare, Japan. Clin Neurol Neurosurg 1997;99:S238.

17. Ohta T, Tanaka H, Kuroiwa T. Diffuse lep- tomeningeal enhancement, ivy sign, in magnetic resonance images of moyamoya disease in childhood: case report. Neurosurgery 1995;37:1009.

18. Roach ES, Golomb MR, Adams R. Management of stroke in infants and children: a scientific statement from a Special Writing Group of the American Heart Association Stroke Council and the Council on Cardiovascular Disease in the Young. Stroke 2008;39:2644.

19. Miyawaki S, Imai H, Takayanagi S. Identification of a genetic variant common to moyamoya disease and intracranial major artery stenosis/occlusion. Stroke 2012;43:3371.

20. Yamamoto M, Aoyagi M, Tajima S. Increase in elastin gene expression and protein synthesis in arterial smooth muscle cells derived from patients with moyamoya disease. Stroke 1997;28:1733. 